\title{
Effect of Kuntai Capsule Combined with Nilestriol Tablets in the Treatment of Perimenopausal Syndrome and on Reproductive Hormones
}

\author{
CAIYAN XU, XIULAN TIAN* AND JIANJUN ZHAI \\ Department of Gynecology and Obstetrics, Beijing Tongren Hospital, Capital Medical University, Beijing 100176, China
}

XU et al.: Efficacy of Kuntai Capsule Combined with Nilestriol Tablets

\begin{abstract}
To investigate the efficacy of the Kuntai capsules combined with nilestriol tablets in the treatment of perimenopausal syndromes and on reproductive hormones, a total of 108 patients with perimenopausal syndromes detected by clinical diagnosis in the department of gynecology and obstetrics in Beijing Tongren Hospital were enrolled from January 2018 to June 2019. These clinical subjects were randomly divided into the observation group $(n=54)$ and control group $(n=54)$. The patients in the control group were treated with nilestriol $2 \mathrm{mg}$ once every $2 \mathrm{w}$ and the dosage was changed to $1 \mathrm{mg}$, twice a month after symptom improvement. The patients in the observation group were treated with oral Kuntai capsules in addition to nilesteriol, 4 each time, twice a day. Both groups were treated continuously for 3 mouths. The curative effect, clinical symptom score and reproductive hormone level before and after treatment were compared between the two groups. The results showed that the Kupperman menopause index and hot flush scores of both groups were significantly decreased from baseline levels. In the 3 mouths after treatment, the Kupperman menopause index in the observation group was lower than that in the control group ( $(p=0.031)$, but there was no significant difference after 6 mouths $(p=0.178)$. In the observation group, the hot flush score was significantly lower than that of the control group $(p=0.05)$. The results showed that after treatment, the levels of reproductive hormones, follicle stimulating hormone, luteinizing hormone and testosterone and blood lipids, Triglyceride, total cholesterol, low-density lipoprotein cholesterol and high-density lipoprotein cholesterol in the two groups changed significantly compared to those before treatment. The levels of follicle stimulating hormone, luteinizing hormone, total cholesterol and lowdensity lipoprotein cholesterol in the observation group were lower than those in the control group, while the levels of serum estradiol and high-density lipoprotein cholesterol in the observation group were higher than those in the control group. Kuntai capsule may be a good alternative therapy for patients who have contraindication or concerns of hormone therapy.
\end{abstract}

Key words: Nilestriol tablets, kuntai capsules, perimenopausal syndrome, Kupperman menopause index, perimenopausal hot flush

The perimenopausal period was an important physiological transition period, which was characterized by the decline of the ovarian function, and the response of ovary to the gonadotropin (GN) stimulation was gradually decreased, and the secretion of estrogen and progesterone was decreased. The perimenopausal women have a series of physical and psychological diseases, the hot flush, sweating, insomnia, impatience, and fatigue were the commonest symptoms ${ }^{[1]}$. Therefore, in this special period, the life of the women will be better if they can get appropriate medical care. At present, the efficacy of the hormone therapy (HT) in the treatment of perimenopausal syndromes (PMS) has been recognized ${ }^{[2]}$. However, the long-term use of hormones will increase the incidence of endometrial cancer and breast cancer. In addition, some perimenopausal patients have contraindications or precautions for HT. Therefore, it is necessary to develop more effective treatments for the perimenopausal women. Kuntai capsule (control group) was a single Chinese patented drug, which was made from the Coptis chinensis. According to the Treatise on Febrile Diseases, the Kuntai capsule was composed of Phaseolus calcaratus, Radices paeoniae alba, Cornus officinalis, Scutellaria baicalensis, and Poria cocos, which has the functions of nourishing

*Address for correspondence 
yin, clearing heat, and calm the nerves ${ }^{[3]}$. Since it was approved as a clinical drug in 2000 , the Kuntai capsule has been widely and effectively used in the treatment of related diseases such as premenstrual syndrome or ovarian dysfunction. A number of trials have compared the efficacy and adverse reactions of Kuntai capsule and various estrogens on the market in the treatment of the premenstrual syndrome, or their effects on different clinical symptoms ${ }^{[4]}$. However, there was still a lack of systematic evaluation of the efficacy of the Kuntai capsule combined with other drugs. Therefore, this study aims to evaluate the efficacy of the Kuntai capsule combined with Nilestriol tablets in the treatment of PMSs and its effect on reproductive hormones. All patients met the diagnostic criteria of PMSs in Obstetrics and Gynecology ${ }^{[5]}$ and the syndrome differentiation criteria of stagnation of liver-qi type in practical gynecology of Traditional Chinese Medicine ${ }^{[6]}$. Patients have complete clinical data and follow-up records. The patients had no hepatic and renal insufficiency. The patients had no malignant lesions of the uterus and ovary. The patients have no family history of mental illness or mental illness, and can communicate normally. The patients were not allergic to the drugs in this study. The exclusion criteria included those patients with malignant tumor and tuberculosis, patients who have taken contraceptive, lipid-lowering, and anticoagulant drugs recently, patients with serious diseases of the endocrine system and hematological system. A total of 108 patients with PMS diagnosed by clinical diagnosis in the department of obstetrics and gynecology in Beijing Tongren Hospital were enrolled from January 2018 to June 2019 and were randomly divided into the observation group $(n=54)$ and the control group $(n=54)$. Statistical analysis showed that there was no significant difference in age, course of disease, body mass index, serum estradiol (E2), and follicle stimulating hormone (FSH) level between the 2 groups (Table 1). The patients in the control group were treated with nilestriol (Hunan Zhengqing Pharmaceutical Group Co., Ltd.) at $2 \mathrm{mg}$ once every $2 \mathrm{w}$, and the dosage was changed to $1 \mathrm{mg}$, twice a month after symptom improvement. The patients in the observation group were treated with oral Kuntai capsule (Guiyang Xintian Pharmaceutical Co., Ltd., National medicine permission number: Z20000083) in addition to nilestriol tablets, 4 capsules each time, twice a day. Both groups were treated continuously for 3 mouths. According to the clinical characteristics of the premenopausal period (mental exhaustion, insomnia, emotional instability, depression and paranoia, dizziness, arthralgia, and headache), the total score of Kupperman menopause index $(\mathrm{KMI})^{[5]}$, hot flush ${ }^{[6]}$, insomnia ${ }^{[7]}$ were calculated at $0,3,6$, 9, and 12 mo after treatment. KMI score can reflect the severity of the palpitation, labor pains, sexual life, and urinary tract infection in PMSs. The higher score indicates the more serious of the symptoms. Reproductive hormone level were detected using $3 \mathrm{ml}$ of the fasting peripheral venous blood that was collected from all patients before treatment and 3 mouths after treatment and the serum was separated by centrifuging at $3000 \mathrm{rpm}$ for $10 \mathrm{~min}$. The contents of the reproductive hormones including luteinizing hormone (LH), FSH, and E2 were detected by the radioimmunoassay. The results were provided by the laboratory department of Beijing Tongren Hospital. The levels of serum total cholesterol (TC), low-density lipoprotein cholesterol (LDL-C), and high-density lipoprotein cholesterol (HDL-C) were measured by the automatic biochemical analyser (Boko BK -400 automatic biochemical analyser) before treatment and 3 mouths after treatment. The data were analyzed with SPSS 18.0, the clinical symptom score and the levels of the reproductive hormone were in accordance with the normal distribution. Paired t-test was used to compare the sample mean before and after treatment in the same group, group test was used to compare the sample mean between the two groups, and the-rank sum test was used to compare the grade data. $p<0.05$ indicates that the difference is statistically significant. At 3, 6, 9 and $12 \mathrm{t}$ mo after treatment, the KMI in both groups decreased significantly compared with the baseline level $(\mathrm{p}<0.01)$. At the 3rd mo after treatment, the KMI of the observation group was lower than that of the control group $(\mathrm{p}=0.031)$, but there was no significant difference after 6 mouths $(\mathrm{p}=0.0178)$. In addition, the KMI decreased gradually during the 12 mo treatment period, indicating that the hormone or Kuntai capsule could relieve PMS. And the therapeutic effect was a positive correlation to the treatment time. The results showed that the the Kuntai capsule has a good therapeutic effect in short and long-term treatment. There was no significant difference in the score of hot flush at the baseline level $(\mathrm{p}=0.757)$. Compared to the baseline, the incidence of hot flush in the 2 groups

TABLE 1: COMPARISON OF THE CLINICAL DATA BETWEEN TWO GROUPS

\begin{tabular}{lcccc}
\hline $\begin{array}{l}\text { Demographic } \\
\text { data }\end{array}$ & $\begin{array}{c}\text { Observation } \\
\text { group }(\mathrm{n}=54)\end{array}$ & $\begin{array}{c}\text { Control } \\
\text { group }(\mathrm{n}=54)\end{array}$ & $\mathrm{t}$ & $\begin{array}{c}\mathrm{p} \\
\text { value }\end{array}$ \\
\hline Age $(\mathrm{y})$ & $53.66 \pm 3.58$ & $54.29 \pm 3.76$ & 0.357 & 0.635 \\
$\begin{array}{l}\text { Course of } \\
\text { disease }(\mathrm{y})\end{array}$ & $1.26 \pm 0.18$ & $1.44 \pm 0.25$ & 0.213 & 0.793 \\
$\begin{array}{l}\text { Body mass } \\
\text { index }\left(\mathrm{kg} / \mathrm{m}^{2}\right)\end{array}$ & $22.13 \pm 1.28$ & $22.09 \pm 1.12$ & 0.102 & 0.907 \\
\hline
\end{tabular}


decreased sharply after 3 mouths of treatment $(p<0.01)$ and the improvement in the observation group was more obvious $(\mathrm{p}<0.05)$. There were similar results after 6, 9, and 12 mo of treatment (Table 3). These results showed that both Kuntai capsule and nilestriol tablets were effective in the treatment of hot flush, and the Kuntai capsule has a better therapeutic effect. Before treatment, there was no significant difference in the levels of E2, TC, FSH, and LH between the 2 groups ( $>0.05)$. After treatment, the levels of E2 increased in both groups, while the levels of TC, FSH, and $\mathrm{LH}$ decreased in both groups, and the difference was statistically significant $(\mathrm{p}<0.05)$. After treatment, the level of E2 in the observation group was higher than that in the control group, while the levels of T, FSH, and $\mathrm{LH}$ in the observation group were significantly lower than those in the control group $(\mathrm{p}<0.05$, Table 4). Before treatment, there was no statistical difference in the blood lipid indbetween the two groups $(\mathrm{p}>0.05)$. After treatment, the changes of the blood lipid level in the observation group was larger than that in the control group, and the difference was statistically significant $(\mathrm{p}<0.05$, Table 5).

The incidence of PMSs was high, which seriously disturbed the physical and mental health of the middleaged and elderly. However, the middle-aged and elderly pay less attention to PMSs. According to the study, more than $50 \%$ of people in China were troubled by PMSs, but few of these patients seeking medical treatment and receiving treatment ${ }^{[7]}$. An epidemiological study of PMSs in women aged 50-60 in Shanghai ${ }^{[8]}$ showed that $62 \%$ of patients had at least a single perimenopausal symptom, $65 \%$ of patients needed treatment, and $72 \%$ needed PMSs counselling. However, these people have a very low consultation rate. At present, hormonal replacement was the main treatment for PMSs because it can improve the symptoms (hot flush and sweating) and quality of life of the patients with PMSs. However, some patients have contraindications to HT, including mastitis or suspected breast cancer, hysteromyoma or sex-related malignant tumor, thromboembolic disease, and severe renal dysfunction. In recent years, many

TABLE 2: THE KMI SCORES OF THE TWO GROUPS

\begin{tabular}{lcccccc}
\hline \multirow{2}{*}{ Group } & & \multicolumn{5}{c}{ KMI scores } \\
\cline { 3 - 7 } & Cases & Initial & $\begin{array}{c}3 \text { mouths after } \\
\text { treatment }\end{array}$ & $\begin{array}{c}\text { 6 mouths after } \\
\text { treatment }\end{array}$ & $\begin{array}{c}\mathbf{9} \text { mo after } \\
\text { treatment }\end{array}$ & $\begin{array}{c}12 \text { mo after } \\
\text { treatment }\end{array}$ \\
\cline { 3 - 8 } Observation & 54 & $22.67 \pm 6.33$ & $14.12 \pm 3.15$ & $11.09 \pm 2.66$ & $10.08 \pm 1.06$ & $8.28 \pm 0.37$ \\
Control & 54 & $22.89 \pm 7.67$ & $15.88 \pm 3.13^{*}$ & $12.87 \pm 2.19^{*}$ & $11.25 \pm 1.21^{*}$ & $9.95 \pm 0.53^{*}$ \\
\hline
\end{tabular}

*Indicates significant differences between the two groups, $\mathrm{p}<0.05$

TABLE 3: THE HOT FLUSH SCORES OF THE TWO GROUPS

\begin{tabular}{ccccccc}
\hline \multirow{2}{*}{ Group } & Cases & \multicolumn{5}{c}{ KMI scores } \\
\cline { 2 - 7 } & Initial & $\begin{array}{c}\text { 3 mouths after } \\
\text { treatment }\end{array}$ & $\begin{array}{c}\text { 6 mouths after } \\
\text { treatment }\end{array}$ & $\begin{array}{c}\mathbf{9} \text { mo after } \\
\text { treatment }\end{array}$ & $\begin{array}{c}12 \text { mo after } \\
\text { treatment }\end{array}$ \\
\hline Observation & 54 & $6.41 \pm 2.87$ & $2.26 \pm 1.37$ & $2.21 \pm 1.05$ & $2.07 \pm 1.27$ & $1.65 \pm 0.78$ \\
Control & 54 & $6.35 \pm 3.02$ & $3.87 \pm 1.53^{*}$ & $3.26 \pm 1.65^{*}$ & $3.06 \pm 1.54^{*}$ & $2.67 \pm 0.58^{*}$ \\
\hline
\end{tabular}

*Indicates significant differences between the two groups, $p<0.05$

TABLE 4: COMPARISON OF THE REPRODUCTIVE HORMONE LEVELS BETWEEN THE TWO GROUPS BEFORE AND AFTER TREATMENT

\begin{tabular}{|c|c|c|c|c|c|c|c|c|c|}
\hline \multirow[t]{2}{*}{ Group } & \multirow{2}{*}{ Cases } & \multicolumn{2}{|c|}{ E2 (mM) } & \multicolumn{2}{|c|}{$\mathrm{TC}(\mathrm{mM})$} & \multicolumn{2}{|c|}{ FSH (mM) } & \multicolumn{2}{|c|}{ LH (mM) } \\
\hline & & Before & After & Before & After & Before & After & Before & After \\
\hline Observation & 54 & $17.58 \pm 8.62$ & $24.41 \pm 7.65$ & $4.68 \pm 1.51$ & $3.25 \pm 1.116$ & $61.88 \pm 19.08$ & $50.61 \pm 9.24$ & $11.25 \pm 2.81$ & $8.64 \pm 2.58$ \\
\hline Control & 54 & $18.82 \pm 8.81$ & $31.45 \pm 7.48^{*}$ & $4.72 \pm 1.65$ & $1.57 \pm 0.65^{*}$ & $62.20 \pm 19.26$ & $42.10 \pm 8.53^{*}$ & $11.32 \pm 1.98$ & $6.23 \pm 1.10^{*}$ \\
\hline
\end{tabular}

TABLE 5: COMPARISON OF THE BLOOD LIPID LEVELS BETWEEN THE TWO GROUPS BEFORE AND AFTER TREATMENT

\begin{tabular}{|c|c|c|c|c|c|c|c|c|c|}
\hline \multirow{2}{*}{ Group } & \multirow{2}{*}{ Cases } & \multicolumn{2}{|c|}{ TG (mM) } & \multicolumn{2}{|c|}{$\mathrm{TC}(\mathrm{mM})$} & \multicolumn{2}{|c|}{ HDL-C (mM) } & \multicolumn{2}{|c|}{ LDL-C (mM) } \\
\hline & & Before & After & Before & After & & & Before & After \\
\hline $\begin{array}{l}\text { Observation } \\
\text { group }\end{array}$ & 54 & $5.87 \pm 1.42$ & $5.05 \pm 1.12$ & $2.89 \pm 0.96$ & $1.87 \pm 0.42$ & $4.85 \pm 1.08$ & $4.09 \pm 0.91$ & $1.29 \pm 0.31$ & $1.47 \pm 0.16$ \\
\hline Control group & 54 & $5.92 \pm 1.43$ & $4.32 \pm 0.95^{*}$ & $2.84 \pm 0.94$ & $1.12 \pm 0.33^{*}$ & $4.73 \pm 0.91$ & $3.01 \pm 0.38^{*}$ & $1.26 \pm 0.37$ & $1.83 \pm 0.49^{*}$ \\
\hline
\end{tabular}

*Indicates significant differences between the two groups, $\mathrm{p}<0.05$ 
clinicians have applied the Kuntai capsule to PMSs patients and achieved good results ${ }^{[9]}$. Two studies have shown that the Kuntai capsule can relieve sleep, anxiety, and depression in the perimenopausal women effectively ${ }^{[10,11]}$. A randomized, double-blind, parallelcontrolled clinical trial ${ }^{[12]}$ showed that the Kuntai capsule has a similar effect to estradiol valerate in relieving the menopausal symptoms in patients with the menopausal and postmenopausal transition, especially in the postmenopausal transitional patients. It is suggested that the effect of Kuntai capsule on the climacteric syndrome may be related to the ovarian function, Kuntai capsule may improve the ovarian function during climacteric transition. Yang et al. ${ }^{[13]}$ showed that the efficacy of the Kuntai capsule in the treatment of climacteric syndrome was similar to that of estrogen, and had no effect on vital signs, liver and kidney function, and endometrial thickness. Chen et al. ${ }^{[4]}$ pointed out that the Kuntai capsule was more effective than the low-dose estrogen in treating some symptoms (numbness, depression, dizziness, fatigue, muscle and joint pain, headache) of climacteric transition. In addition, a number of clinical trials have confirmed that the Kuntai capsule has a low incidence of adverse reactions and has a similar effect on PMSs and quality of sexual life in the perimenopausal women ${ }^{[14]}$. Kuntai capsule was more effective in improving the blood lipids than hormone therapy, which was good news for women with coronary heart disease.

During menopause, the women accompanied by the deficiency of kidney qi, kidney yin and yang, and the imbalance of yin and yang, which caused the dysfunction of the body ${ }^{[15]}$. According to Suwen Shanggu Tianzhen Lun, when the woman was 49 y old, the Ren channel and Taichong channel were weakened in qi and blood, the radices semiaquilegiae exhausted and the menstruation stopped. It was considered that the disease originates from kidney deficiency and involves the heart, liver, and spleen. Therefore, the principle of treatment should be the calm heart and tranquilizing the mind, invigorating the spleen for eliminating dampness, relieving the depressed liver, and nourishing the liver and kidney ${ }^{[16]}$. The results showed that after treatment, the levels of reproductive hormones (FSH, LH, T) and blood lipids (TC, LDL-C, HDL-C) in the 2 groups changed significantly compared with those before treatment. The levels of FSH, LH, TC, and LDL-C in the observation group were lower than those in the control group, while the levels of E2 and HDL-C in the observation group were higher than those in the control group. It shows that the Kuntai capsule combined with nilestriol tablets can treat the climacteric syndrome effectively, and improve the levels of reproductive hormones and blood lipids significantly, and promote the recovery of patients. Kuntai capsule was a Chinese patent drug evolved from huanglianejiao decoction, which was mainly made of traditional Chinese medicine such as Coptis chinensis, Donkey-hide gelatin, Poria cocos, Scutellaria baicalensis, Prepared Rehmannia root, and radix paeoniae alba. Modern pharmacology shows that the Donkey-hide gelatin can improve the endometrial blood supply and microcirculation, regulate the level of sex hormones in postmenopausal women, increase the level of flavonoids, inhibit the sweat gland secretion, improve insomnia and effectively improve menopausal ovarian function. Prepared Rehmannia root has a phytoestrogenic effect, which can increase the level of HDL-C, reduce the level of LDL-C, promote the cholesterol into the blood and metabolize the liver. It can also remove the cholesterol from the blood vessel wall, decompose lipoprotein and inhibit it, so as to cure both the symptoms and the root causes ${ }^{[17,18]}$.

In conclusion, the Kuntai capsule may be a good alternative therapy for patients who have contraindication or concerns about hormone therapy. Therefore, a double-blind, randomized, controlled, large sample size clinical study should be conducted to further ensure the efficacy and safety of the Kuntai capsule.

\section{Author's contributions:}

Xiulan Tian conceived and designed the experiments; Jianjun Zhai performed the experiments; Caiyan Xu analyzed the data and wrote the paper.

\section{Acknowledgement:}

This work was supported by Beijing Tongren Hospital, Capital Medical University.

\section{Declaration of interest:}

The authors report no conflicts of interest.

\section{REFERENCES}

1. Oldmeadow JA, Dixson BJ. The association between men's sexist attitudes and facial hair. Arch Sex Behav 2016;45:891-9.

2. Dixson BJ, Sulikowski D, Gouda-Vossos A, Rantala MJ, Brooks RC. The masculinity paradox: facial masculinity and beardedness interact to determine women's ratings of men's facial attractiveness. J Evol Biol 2016;29:2311-20.

3. Dixson BJ, Kennedy-Costantini S, Lee AJ, Nelson NL. Mothers are sensitive to men's beards as a potential cue of paternal investment. Horm Behav 2019;113:55-66.

4. Sijpesteijn PM. Beards, Braids, and Moustachios: Exploring 
the Social Meaning of Hair in the Mediaeval Muslim World. Al-Masāq 2018;30:4-8.

5. Ingprasert $\mathrm{S}$, Tanglertsampan $\mathrm{C}$, Tangphianphan $\mathrm{N}$, Reanmanee C. Efficacy and safety of minoxidil $3 \%$ lotion for beard enhancement: A randomized, double-masked, placebocontrolled study. J Dermatol 2016;43:968-9.

6. Saeedi M, Kosaryan M, Fekri K, Adabi M, Mahdavi MR. A randomized, double-blind, controlled trial of testosterone gel treatment versus vehicle control on the facial hair of young men with beta-thalassemia major. J Dermatolog Treat 2007;18:271-4.

7. Vestita M, Giudice G, Bonamonte D, Apruzzi D, Filoni A. Beard hair density increase. A possible role of topical tretinoin application?. Dermatol Ther 2016;30.

8. Suchonwanit P, Thammarucha S, Leerunyakul K. Minoxidil and its use in hair disorders: a review. Drug Des Devel Ther 2019;13:2777-86.

9. Chellini PR, Pirmez R, Raso P, Sodre CT. Generalized hypertrichosis induced by topical minoxidil in an adult woman. Int J Trichology 2015;7:182-3.

10. Rampon G, Henkin C, de Souza PR, Almeida HL Jr. Infantile generalized hypertrichosis caused by topical minoxidil. An Bras Dermatol 2016;91:87-8.

11. Lee S, Tanglertsampan C, Tanchotikul M, Worapunpong N. Minoxidil $2 \%$ lotion for eyebrow enhancement: A randomized, double-blind, placebo-controlled, spilt-face comparative study. J Dermatol 2014;41:149-52.

12. Suwanchatchai W, Tanglertsampan C, Pengsalae N, Makornwattana M. Efficacy and safety of bimatoprost 0.03 $\%$ versus minoxidil $3 \%$ in enhancement of eyebrows: a randomized, double-blind, split-face comparative study. J Dermatol 2012;10:865-6.

13. Han JH, Kwon OS, Chung JH, Cho KH, Eun HC, Kim KH. Effect of minoxidil on proliferation and apoptosis in dermal papilla cells of human hair follicle. J Dermatol Sci 2004;34:918.

14. Lachgar S, Charveron M, Gall Y, Bonafe JL. Minoxidil upregulates the expression of vascular endothelial growth factor in human hair dermal papilla cells. Br J Dermatol 1998;138:407-11.

15. Itami S, Sonoda T, Kurata S, Takayasu S. Mechanism of action of androgen in hair follicles. J Dermatol Sci 1994;7:S98-103.

16. Thornton MJ, Hamada K, Messenger AG, Randall VA. Androgen-dependent beard dermal papilla cells secrete autocrine growth factor(s) in response to testosterone unlike scalp cells. J Invest Dermatol 1998;111:727-32.

17. Giltay EJ, Gooren LJ. Effects of sex steroid deprivation/ administration on hair growth and skin sebum production in transsexual males and females. J Clin Endocrinol Metab 2000;85:2913-21.

18. Bazzano GS, Terezakis N, Galen W. Topical tretinoin for hair growth promotion. J Am Acad Dermatol 1986;15:880-93.

19. Bazzano G, Terezakis N, Attia H, Bazzano A, Dover R, Fenton $\mathrm{D}$, et al. Effect of retinoids on follicular cells. J Invest Dermatol 1993;101:S138-42.

20. Kmiec ML, Pajor A, Broniarczyk-Dyła G. Evaluation of biophysical skin parameters and assessment of hair growth in patients with acne treated with isotretinoin. Postepy Dermatol Alergol 2013;30:343-9.

21. İslamoglu ZG, Altınyazar HC. Effects of isotretinoin on the hair cycle. J Cosmet Dermatol 2019;18:647-51.

22. Yoo HG, Chang IY, Pyo HK, Kang YJ, Lee SH, Kwon OS, et al. The additive effects of minoxidil and retinol on human hair growth in vitro. Biol Pharm Bull 2007;30:21-6.

23. Kwon OS, Pyo HK, Oh YJ, Han JH, Lee SR, Chung JH, et al. Promotive effect of minoxidil combined with all-trans retinoic acid (tretinoin) on human hair growth in vitro. J Korean Med Sci 2007;22:283-9.

24. Ferry JJ, Forbes KK, VanderLugt JT, Szpunar GJ. Influence of tretinoin on the percutaneous absorption of minoxidil from an aqueous topical solution. Clin Pharmacol Ther 1990;47:43946.

25. Sharma A, Goren A, Dhurat R, Agrawal S, Sinclair R, Trueb RM, et al. Tretinoin enhances minoxidil response in androgenetic alopecia patients by upregulating follicular sulfotransferase enzymes. Dermatol Ther 2019;32:e12915.

26. Buhl AE, Waldon DJ, Baker CA, Johnson GA. Minoxidil sulfate is the active metabolite that stimulates hair follicles. $\mathrm{J}$ Invest Dermatol 1990;95:553-7.

27. Goren A, Shapiro J, Roberts J, McCoy J, Desai N, Zarrab, et al. Clinical utility and validity of minoxidil response testing in androgenetic alopecia. Dermatol Ther 2015;28:13-6.

28. Civas E, Aksoy B, Aksoy HM, Calikoglu E. Follicular unit extraction as a valuable method for the restoration of beard: Retrospective case series study. J Cosmet Dermatol 2020;19:2049-56.

This is an open access article distributed under the terms of the Creative Commons Attribution-NonCommercial-ShareAlike 3.0 License, which allows others to remix, tweak, and build upon the work non-commercially, as long as the author is credited and the new creations are licensed under the identical terms

\begin{tabular}{l}
$\begin{array}{l}\text { This article was originally published in a special issue, } \\
\text { "Biomedical Research in Clinical and Preclinical } \\
\text { Pharmaceutics" Indian J Pharm Sci 2020:82(3)Spl issue7;26-30 }\end{array}$ \\
\hline
\end{tabular}

